\title{
Chronic Osteomyelitis of the Maxilla and Mandible: Microbiological and Clinical Aspects
}

\author{
Osteomielitis Crónica del Maxilar y Mandíbula: Aspectos Microbiológicos y Clínicos
}

\begin{abstract}
Elerson Gaetti-Jardim Júnior; Francisco Isaak Nicolas Ciesielski; Ricélia Possagno; Alvimar Lima de Castro; Antonio Carlos Marqueti \& Ellen Cristina Gaetti-Jardim
\end{abstract}

GAETTI-JARDIM, E. JR.; CIESIELSKI, F, I, N.; POSSAGNO, R.; CASTRO, A. L.; MARQUETI, A. C. \& GAETTI-JARDIM. Chronic osteomyelitis of the maxilla and mandible: microbiological and clinical aspects. Int. J. Odontostomat., 4(2):197202, 2010.

\begin{abstract}
: osteomyelitis of the mandible and maxilla are common in developing countries and their treatment may be long-standing and difficult. Thus, the aim of this study was to discuss the main biological aspects of the chronic osteomyelitis of the jaws of especial interest for dental team. These infections are associated with a complex microbiota composed mainly by anaerobic bacteria, sometimes associated with microorganisms originated from the skin and digestive tract. These data suggest that chronic osteomyelitis of the mandible and maxilla should be treated as anaerobic infections in most cases. In addition, local surgical treatments are relevant in the therapy outcome, associated to the use of antimicrobial agents, and the failure to accomplish them is a major cause of treatment failure.
\end{abstract}

KEY WORDS: osteomyelitis, bacteria, anaerobes, maxilla, mandible.

\section{INTRODUCTION}

Secondary chronic osteomyelitis of the jaws are common in the developing countries, where these diseases are associated with trauma, surgical procedures and previous infections, such as endodontic and periapical infections. However, their incidence, clinical characteristics and etiology have not been studied in details (Prasad et al., 2007). Among the skull bones, the chronic osteomyelitis is more frequently observed in the mandible and, in fewer cases, in the maxilla, but generally limited to one anatomic site (Baltensperger et al., 2004; Lew \& Waldvogel, 2004), although it may spread out to other areas, specially in diabetic, immunosuppressed and hospitalized patients (Brady et al., 2006).

The pathogenesis of these diseases may be linked to hematogenous dissemination of exogenous or commensal microorganisms living on the skin or in the digestive tract, but generally the main source of microorganisms involved in the osteomyelitis of the maxilla and mandible is the dental biofilm and oral infections, particularly endodontic infections (Brady et al., 2006), peri-implantitis, periodontitis and gingivitis (O'Sullivan et al., 2006; Coviello \& Stevens, 2007).

The treatment of these infections depends on the sequestrectomy, surgical debridement of the wound, and removal of the bone cortex, associated to the systemic use of antimicrobials (Lew \& Waldvogel 2004; Coviello \& Stevens, 2007; Brook, 2008b). However when the clinician is not able to proceed the local surgical treatment or due to the microbiota associated to the infectious process (Brook, 2008b) or anatomic and/tissue peculiarities, sometimes the antimicrobial drugs first prescribed are not efficient, making the microbiological diagnosis even more critical, once this diagnosis must dictate which drug should be used (Kim \& Jang 2001; Coviello \& Stevens, 2007). In addition, most microorganisms involved in the pathogenesis of osteomyelitis of the jaws are strict 
anaerobes, many of them fastidious and very difficult to cultivate (Kim \& Jang, 2001; Coviello \& Stevens, 2007, Brook, 2008b).

\section{CLINICAL AND RADIOGRAPHIC ASPECTS}

Although the age does not have a major role on the incidence of maxillary osteomyelitis, it is more frequent in adolescents and adults between 40 and 60 years old (Baltensperger et al., 2004). The chronic maxillary osteomyelitis may be classified into two major groups: primary chronic osteomyelitis, where the disease always evidenced chronic symptoms and secondary chronic osteomyelitis, which is the evolution of a previous acute osteomyelitis or the local dissemination of chronic odontogenic infections. Primary chronic osteomyelitis has an insidious beginning and clinical and radiographic manifestations can take weeks to become evident (Kim \& Jang, 2001), whereas acute osteomyelitis generally takes 4 weeks to become a chronic infection (Lew \& Waldvogel, 2004).

In the last fifteen years, forty-five patients with secondary chronic osteomyelitis of the jaws were seen at School of Dentistry of Araçatuba, Brazil. Long-standing localized bone pain was observed in $68.9 \%$, edema $(37.4 \%)$, purulent secretion $(31.1 \%)$ osteolytic lesions (82.2\%), bone condensation (91.1\%), and bone sequestra $(64.4 \%)$ are the most common clinical and radiographic signs. Although not frequent, intraoral fistula (8.9\%), extraoral fistula (4.4\%; Fig. 1), erythema $(4.4 \%)$, periosteal thickening and bony irregularities $(8.9 \%)$ were detected, particularly in young patients, as also reported in literature (Kim \& Jang, 2001; Baltensperger et al., 2004). In $26,7 \%$, osteomyelitis was associated to an iatrogenic dental procedure, generally after fracture of mandible (Fig. 2) or endodontic treatment.

The radiographic characteristics of the osteolysis observed in the chronic osteomyelitis of the maxilla and mandible are more evident in younger patients; meanwhile the bone condensation is more frequent in adults, particularly in lesions of the mandible. However, these characteristics are often modified by selfmedication with anti-inflammatory and antimicrobial drugs, making the diagnosis even harder. Moreover, these clinical and radiographic signs may vary according to the predisposing factor, such as odontogenic infections, bone and tooth fractures, maligning neoplasia, use of bisphosphonates, systemic lupus, multiple myeloma and radiotherapy for head and neck cancer (Prasad et al., 2007; Rush et al., 2007; Brook, 2008b).
Although the mandible is the bone most frequently affected by chronic osteomyelitis, the factors associated with this phenomenon remain unclear. This increased frequency of osteomyelitis in the mandible also occurs in other animal species such as dogs, cats and marine mammals. It is probable that the mandibular bone structure, less plastic than the maxillary bone, may allow microbial persistence, as well as the increased vulnerability of the mandible may be related to trauma (Sharkawy, 2007) and higher incidence of predisposing factors such as pericoronitis (Betts et al., 1996). Other peculiarities of the mandibular bone include exuberant peripheral reactive bone formation, while this feature is rare in the maxilla (Betts et al., 1996).

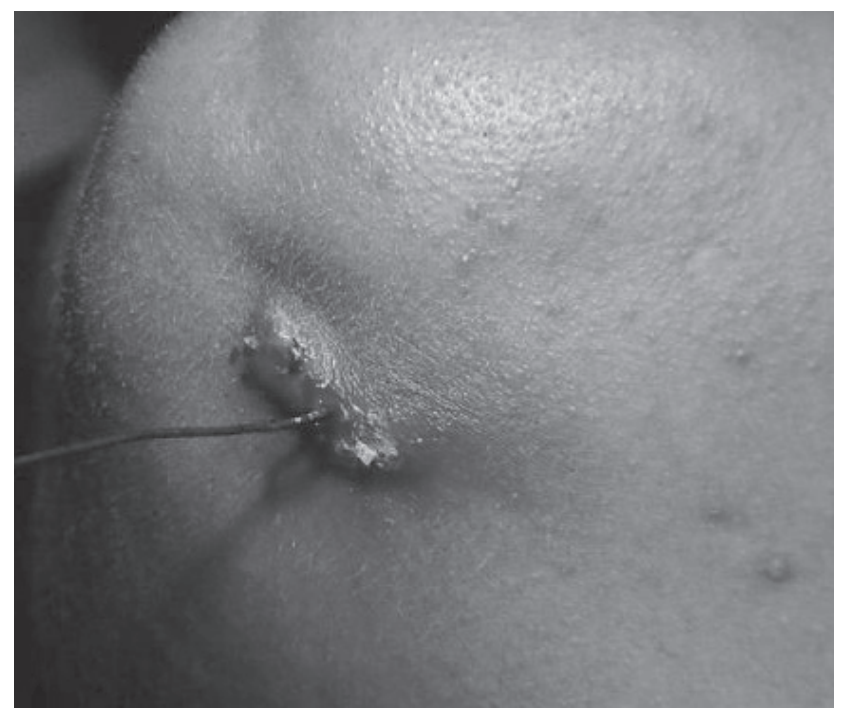

Fig. 1. Extraoral fistula in a mentally disabled person, who had the habit of nudging the mandible fistula with the aid of a small wire, causing skin and environment bacteria to penetrate the mandibular lesion.

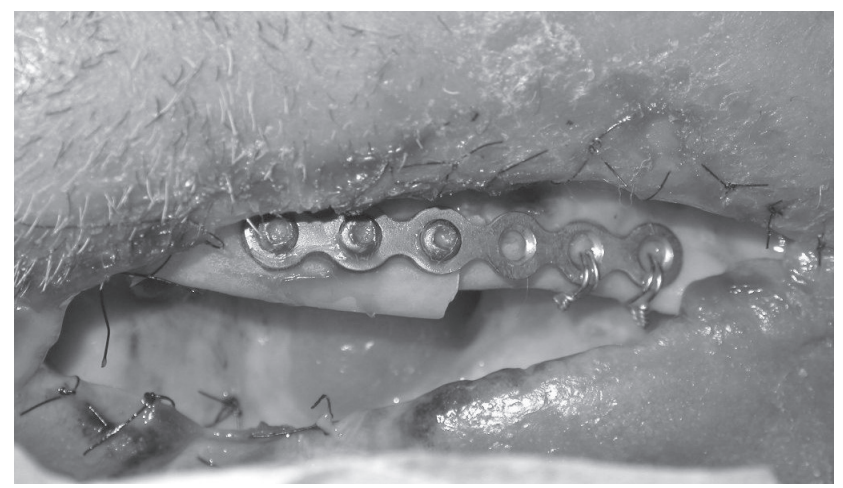

Fig. 2. A clinical case presenting a more acute infection associated with iatrogenic injury by a surgeon, who made the reduction of the mandibular fracture improperly and used a wide range of betalactams in the treatment, without adequate results. 
GAETTI-JARDIM, E. JR.; CIESIELSKI, F, I, N.; POSSAGNO, R.; CASTRO, A. L.; MARQUETI, A. C. \& GAETTI-JARDIM. Chronic osteomyelitis of the maxilla and mandible: microbiological and clinical aspects. Int. J. Odontostomat., 4(2):197-202, 2010.

\section{MICROBIOLOGICAL ASPECTS}

The characteristics of the microbiota associated to the maxillary osteomyelitis depend on the origin of the infectious process: hematogenous origin or direct extensions from oral infectious processes. In the first case, it is notorious the relevance of tolerant microorganisms, such as enteric rods and staphylococci, meanwhile in the second condition the microbiota of the osteomyelitis will depend on the microbiota of the previous infectious process and where it took place (Brook, 2008b). The frequency of the hematogenous osteomyelitis in the jaws is unknown, although bacteremia associated to trauma, dental procedures and tooth brushing is common.

In the maxilla and mandible, the cases of chronic osteomyelitis are polymicrobial and the major sources of the infecting microorganisms are odontogenic, producing an average of 2.4 to 3.9 strict anaerobic species and 0.4 to 1.3 aerobic or facultative anaerobic species per lesion (Brook, 1986). The most commonly detected oral microorganisms in osteomyelitis are represented by Gram-negative anaerobic rods, most of them considered periodontal pathogens, and facultatively anaerobic cocci of genera Staphylococcus and Enterococcus.

The role played by the oral streptococci in chronic osteomyelitis of the jaws is not essential (Zuluaga et al., 2006; Brook, 2008b), but these microorganisms probably provide suitable environmental conditions for the establishment of strict anaerobes in the bone (Baltensperger et al., 2004). In patients with a history of dental trauma or fracture of the jaws, as well as misuse of antimicrobial drugs, the presence of staphylococci and enteric rods and cocci, particularly Enterococcus faecalis, must be considered (Scolozzi et al., 2005; Zuluaga et al., 2006; Brook, 2008b).

Some cases of chronic osteomyelitis of the jaws are tolerant to the antimicrobial treatment and actinomycetes are frequently detected in these situations. The genus Actinomyces is composed by Gram-positive aerotolerant rods, sometimes resistant to antimicrobial drugs (Brook, 2008b), and A. israelii is the most relevant species, particularly to the head and neck infections (Sharkawy, 2007). Some evidences suggest the participation of Actinomyces spp. and Propionibacterium acnes in the etiology of diffuse sclerosing osteomyelitis of the mandible (Baltensperger et al., 2004).

The presence of putrid odor is generally associated with strict anaerobes of the genera Fusobacterium, Porphyromonas, Prevotella and Parvimonas, which may invade actively the tissues through the production of proteases and exopeptidases and can survive and proliferate within osteolytic lesions with poor blood supply (Brook, 2008a).

\section{SAMPLE COLLECTION FOR MICROBIOLOGICAL ANALYSES}

In order to determine microbial etiology of the osteomyelitis of the jaws and the susceptibility to antimicrobials, the first step is the sample collection. The Gram staining can collaborate to determine the bacterial morphotypes present in bone lesion and could guide the selection of antimicrobial therapy.

Although conventional procedures, such as culture, show inferior sensitivity when compared to molecular methods as PCR, culture allows the determination of microbial susceptibility patterns. For microbial diagnosis of chronic osteomyelitis, only bone biopsy, bone sequestra, bone marrow, granulation tissue and aspirated pus specimens are acceptable for microbial diagnosis. In order to avoid cross-contamination from oral microorganisms, the clinical specimens must be collected by surgical procedures, avoiding contact with oral tissues, oral environment and sinus tracts (Prasad et al., 2007). When bone biopsies were removed, areas of the margin of the osteolytic lesion must be preferred.

For the isolation of anaerobic organisms, some pre-reduced media for transportation of the specimens, such as the VMGA III or RTF must be employed. Aspiration of softened septic contents of bone lesion with syringes is adequate to avoid deleterious contact of the sample with oxygen. The inobservance of these details, as well as inadequate conditions of handling and cultivation, determines the high frequency of osteomyelitis of the jaws apparently sterile. However, even in ideal conditions, the frequency of positive cultures may be very modest (Baltensperger et al., 2004), since most patients present long standing infections and antimicrobials by self-medication and inappropriate prescription are common.

In addition, when molecular detection of pathogens is feasible or desirable, the most important concern is where to get the specimens in the bone lesion to avoid contact with oral environment, since PCR and real-time PCR have high sensitivity and false positive results could arise after accidental contamination of the sample. 


\section{TREATMENT}

The occurrence, type, severity and prognostic of the chronic osteomyelitis of the jaws depend on many factors, including the composition and virulence of the microbiota, immunologic response of the host, extension and severity of the infection, as well as the source of the infection (Brady et al., 2006). The analyses of the literature as well as the experience in the isolation and characterization of oral microorganisms suggest that the chronic osteomyelitis of the jaws must be treated as a mixed anaerobic infection most of the time, but when facing a previous use of antimicrobial drugs, surgical procedures or extended trauma, the possibility of the participation of microorganisms from the skin, external environment or digestive tract must be taken in account.

In general, osteomyelitis receive symptomatic treatment besides the immobilization of the maxilla in cases of fractures, oral or intravenous antimicrobial treatment for 4 to 8 weeks, with an average of duration of 31 days (Brook, 2008b), besides the surgical removal of the necrotic tissue and the septic containing drainage, and primary chronic osteomyelitis is much more refractory to antimicrobial and surgical treatments than secondary chronic osteomyelitis.

Osteomyelitis is a surgical disease, complete surgical debridement and not a particular antibiotic regimen is the most important factor for a successful outcome. In these cases, data about previous use of antimicrobial agents is very relevant, since these drugs may affect the susceptibility of the microorganisms associated to head and neck infections, especially osteomyelitis, where the treatment lasts for weeks (Coviello \& Stevens, 2007; Brook., 2008b).

For patients seen at School of Dentistry of Araçatuba, after sample collection, the initial treatment consists of sequestrectomy and antibiotic therapy. When patient has not a history of previous use of blactams, amoxicillin or cephalexin is prescribed for 3-4 weeks (500 mg, three times daily, orally). Other b-lactams, such as other cephalosporins and carbapenems, are also good therapeutic options (Mandracchia et al., 2004), although the number of isolates of the genera Fusobacterium, Porphyromonas and Prevotella producing blactamases is significantly increasing (Handal et al., 2004; Al-Haroni et al., 2008), besides the staphylococci, resistant to almost all available drugs (Brook, 2008b).
However, for patients with history of previous use of amoxicillin and/or other b-lactams, clindamycin is the choice $(600 \mathrm{mg}$ twice daily, orally, during 30 to 60 days). In refractory cases due to presence of enteric Gram-negative rods and anaerobes, additional hyperbaric oxygen therapy (HBO) associated with initial intravenous treatment with ciprofloxacin (100 mg twice daily) and clindamycin (600 mg twice daily) for one week, followed by oral antibiotic therapy with these drugs for four weeks is the standard procedure (Scolozzi et al., 2005). Treatment outcomes must be evaluated at 6 -month intervals within at least 18-month period.

Clindamycin is frequently used in the treatment of anaerobic infections associated with oral microorganisms, evidencing excellent efficiency in bone infections and oral bioavailability. In addition, selfmedication using this drug is rare (Lew \& Waldvogel, 2004; Le Moal et al., 2005) and is an appropriate antibiotic for most Gram-positive bacteria, including some strains of community-acquired methicillin-resistant Staphylococcus aureus (Gutierrez, 2005). If communityacquired methicillin-resistant Staphylococcus aureus is a concern (local rates of MRSA are 5\%-10\%), intravenous treatment with vancomycin or clindamycin is indicated (Gutierrez, 2005).

The Gram-negative anaerobic microorganisms resistant to traditional â-lactams are sensitive to the association of these drugs and â-lactamases inhibitors, such as the clavulanic acid, sulbactam or tazobactam, and metronidazol, for instance. However, metronidazol is not recommended as antimicrobial monotherapy, since most of Gram-positive anaerobes are tolerant and almost all facultative anaerobes are naturally resistant to this drug. (Gaetti-Jardim et al., 2007). Then, metronidazol must be reserved to be used in association with other drugs, particularly â-lactams, especially for the most acute cases.

\section{CONCLUSIONS}

Chronic osteomyelitis of maxilla and mandible are associated to previous oral infections and their microbiota consists primarily of oral microorganisms, especially anaerobes, most of them susceptible to blactams and clindamycin. In addition, the surgical procedures also acquire relevance in the treatment and failure to accomplish them is a major cause of treatment failure. 
GAETTI-JARDIM, E. JR.; CIESIELSKI, F, I, N.; POSSAGNO, R.; CASTRO, A. L.; MARQUETI, A. C. \& GAETTI-JARDIM. Chronic osteomyelitis of the maxilla and mandible: microbiological and clinical aspects. Int. J. Odontostomat., 4(2):197-202, 2010.

GAETTI-JARDIM, E. JR.; CIESIELSKI, F, I, N.; POSSAGNO, R.; CASTRO, A. L.; MARQUETI, A. C. \& GAETTI-JARDIM. Osteomielitis crónica del maxilar y mandíbula: aspectos microbiológicos y clínicos. Int. J. Odontostomat., 4(2):197-202, 2010

RESUMEN: La osteomielitis maxilar y mandibular son comunes en países en desarrollo y su tratamiento puede ser difícil y de larga duración. Así, el objetivo del presente estudio fue discutir los principales aspectos biológicos de especial interés odontológico de la osteomielitis crónica del maxilar. Estas infecciones están asociadas a una microbiota compleja compuesta principalmente de bacterias anaerobias, algunas veces asociadas a microorganismos oriundos de la piel y del tracto digestivo. Estos datos sugieren que la osteomielitis crónica maxilar y mandibular deben ser tratadas como infecciones anaerobias en la mayoría de los casos. Además, tratamientos quirúrgicos locales son relevantes en el éxito de la terapia, asociados al uso de agentes anti-microbianos, e cuyos resultados no satisfactorios representan una de las principales causas de un tratamiento fallo o no adecuado.

PALABRAS CLAVE: Osteomielitis, bacteria, anaerobios, maxilar superior, mandíbula.

\section{REFERENCES}

Al-Haroni, M.; Skaug, N.; Bakken, V. \& Cash, P. Proteomic analysis of ampicillin-resistant oral Fusobacterium nucleatum. Oral Microbiol. Immunol., 23:36-42, 2008.

Baltensperger, M.; Grätz, K.; Bruder, E.; Lebeda, R.; Makek, M. \& Eyrich, G. Is primary chronic osteomyelitis a uniform disease? Proposal of a classification based on a retrospective analysis of patients treated in the past 30 years. J. CranioMaxillofac. Surg., 32:43-50, 2004.

Betts, N. J.; Abaza, N. A. \& Kazemi, A. An expansile bony lesion of the posterior mandible in a 12, yearold girl. J. Oral Maxillofac. Surg., 54:203-9, 1996.

Brady, B. A.; Leid, J. G.; Costerton, J. W. \& Shirtliff, M. E. Osteomyelitis: clinical overview and mechanisms of infection persistence. Clin. Microbiol. Newsletter, 28:65-72, 2006.

Brook, I. Actinomycosis: diagnosis and management. Southern Med. J., 101:1019-23, 2008a.

Brook, I. Anaerobic osteomyelitis in children. Pediatr. Infect. Dis., 5:550-6, 1986.

Brook, I. Microbiology and management of joint and bone infections due to anaerobic bacteria. $J$. Orthop. Sci, 13:160-9, 2008b.

Coviello, V. \& Stevens, M. R. Contemporary concepts in the treatment of chronic osteomyelitis. Oral Maxillofacial. Surg. Clin. North Amer., 19:523-34, 2007.
Gaetti-Jardim, E. Jr.; Landucci, L. F.; Lins, S. A.; Vieira, E. M. \& Oliveira, S. R. Susceptibility of strict and facultative anaerobes isolated from endodontic infection to metronidazole and beta-lactams. J. Appl. Oral Sci., 15: 539-45, 2007.

Gutierrez, K. Bone and joint infections in children. Pediatr. Clin. North Amer., 52:779-94, 2005.

Handal, T.; Olsen, I.; Walker, C. B.; \& Caugant, D. A. bLactamase production and antimicrobial susceptibility of subgingival bacteria from refractory periodontitis. Oral Microbiol. Immunol., 19:303-8, 2004.

Kim, S-G. \& Jang, H-S. Treatment of chronic osteomyelitis in Korea. Oral Surg. Oral Med. Oral Pathol. Oral Radiol. Endod., 92:394-8, 2001.

Le Moal, G.; Juhel, L.; Grollier, G.; Godet, C.; Azais, I. \& Roblot, F. Vertebral osteomyelitis due to Fusobacterium species: report of three cases and review of the literature. J. Infect., 51:e5-e9, 2005.

Lew, D. P. \& Waldvogel, F. A. Osteomyelitis. Lancet, 364:369-79, 2004.

Mandracchia, V. J.; Sandres, S. M.; Jaeger, A. J. \& Nickles, W. A. Management of osteomyelitis. Clin. Pediatr. Med. Surg., 21:335-51, 2004.

O'Sullivan, D.; King, P. \& Jagger, D. Osteomyelitis and pathological mandibular fracture related to a late implant failure: a clinical report. J. Prosthet. Dent., 9:106-10, 2006. 
GAETTI-JARDIM, E. JR.; CIESIELSKI, F, I, N.; POSSAGNO, R.; CASTRO, A. L.; MARQUETI, A. C. \& GAETTI-JARDIM. Chronic osteomyelitis of the maxilla and mandible: microbiological and clinical aspects. Int. J. Odontostomat., 4(2):197-202, 2010.

Prasad, K. C.; Prasad, S. C.; Mouli, N. \& Agarwal, S. Osteomyelitis in the head and neck. Acta Otolaryngol., 127:194-205, 2007.

Rush, D. E.; Abdel-Haq, N.; Zhu, J-F.; Aamar, B. \& Malian, M. Clindamycin versus unasyn in the treatment of facial cellulites of odontogenic origin in children. Clin. Pediatr., 46:154-9, 2007.

Scolozzi, P.; Lombardi, T.; Edney, T. \& Jaques, B. Enteric bacteria in mandibular osteomyelitis. Oral Surg. Oral Med. Oral Pathol. Oral Radiol. Endod., 99:42-6, 2005.

Sharkawy, A. A. Cervicofacial actinomycosis and mandibular osteomyelitis. Infect. Dis. Clin. North Amer., 21:543-56, 2007.

Zuluaga, A. F.; Galvis, W.; Saldarriaga, J. G.; Agudelo, M.; Salazar, B. E. \& Vesga, O. Etiologic diagnosis of chronic osteomyelitis. Arch. Intern. Med., 166:95100, 2006.
Correspondence to:

Elerson Gaetti-Jardim Júnior

Department of Pathology and Oral Diagnostics

School of Dentistry of Araçatuba

São Paulo State University-UNESP

R. José Bonifácio 1193, 16015-050

Araçatuba, SP.

BRAZIL

Tel/fax: +55-18-3636-2797/+55-18-3636-4125.

Email address: egaettij@foa.unesp.br

Received: 20-07-2009

Accepted: $18-05-2010$ 